\title{
SUR LES TROUBLES SENSITIFS ET SENSORIELS OBSERVESS AFRES LOBOTOMIE OU TOPECTOMIE PRÉFRONTALES
}

\author{
Robert Messimy
}

$\mathrm{Si}$ les relations des lobes préfrontaux avec les noyaux dorso-médians du thalamus sont actuellement un fait généralement admis, et si l'on tient compte du rôle majeur attribué à l'intégration thalamo-corticale des perceptions sensitives et sensorielles, il n'est pas étonnant que l'on puisse constater des modifications de ces perceptions après une intervention préfrontale. Au cours des tumeurs frontales, certains auteurs, de la notoriété de K. Goldstein, de Clovis Vincent, avaient attiré l'attention sur les phénomènes d'ordre sensitif et sensoriel; ceux-ci restaient cependant d'interprétation assez obscure.

Dès 1937, nous avions été surpris, au cours de recherches expérinentales sur le singe, par l'importance et la constance des réactions engendrées par les stimulations élémentaires et, par la suite, nous avons cherché à retrouver chez l'homme des faits comparables, d'abord chez des sujets ayant subi une lobectomie par tumeur (notamment avec W. German, en 1938), puis chez les malades soumis à la psycho chirurgie.

Les singes privés des lobes préfrontaux réagissent d'une manière excessive à diverses stimulations; ils deviennent hyperesthésiques à la douleur, à la pression des nerfs, au chaud et au froid. L'excitabilité sensorielle est également accrue; les animaux présentent le réflexe d'aveuglement à la lumière avec du larmoiement, ils réagissent exagérément au bruit. Ces symptômes coïncident avec des troubles paresthésiques d'un type particulier: parfois, les singes ont de très brusques mouvements des mains, comme s'ils avaient reçu une légère décharge électrique; ils se grattent avec une fréquence anormale, spécialement les extrémités, les oreilles, le nez.

Ces troubles persistent, semble-t-il, d'une manière permanente. Ils présentent une certaine analogie avec les troubles sensitifs produits par Dusser de Barenne et $O$. Sager, après injection de très faibles doses de strychnnine dans le thalamus du chat et du singe.

Cette surréactivité existait chez tous nos animaux; chez l'un cependant, qui présentait des états cataleptiques intenses, on observait une curieuse dissociation des troubles sensitifs; lorsqu'il devenait cataleptique, l'animal ne réagissait plus aux stimulations provoquées; à cet état cataleptique, succédaient des crises d'excitation hystériforme où tout examen était impossible. 
Chez l'homme, il est possible de retrouver des troubles sensitits analogues, soit au cours des lésions frontales, comme nous y avons insisté après avoir fait la revue des 172 observations de tumeurs frontales du Dr. Harvey Cushing, soit après lobectomie préfrontale. Ces troubles sont très comparables à ceux qu'on observe après une intervention psycho-chirurgicale, ainsi nous n'y insisterons pas ici.

Dans l'important lot de cas psycho-chirurgicaux de la clinique neurochirurgicale de la Pitié, nous avons conservé pour cette étude 50 ohservations valables, dont 30 lobotomies exécutées par le Professeur Petit-Dutaillis suivant le procédé de Poppen (sauf un cas où la méthode latérale fut utilisée) et 20 topectomies, réalisées pour la plupart par le Dr. Feld. Dans tous ces cas, un examen neurologique pré et post-opératoire put être réalisé.

Dans notre matériel d'étude, nous relevons: 1) 20 malades opérés pour des algies irréductibles, dont 14 d'origine cancéreuse et 6 d'origine diverse (deux algies faciales, une hémialgie post-commotionnelle, une algie tabétique, un moignon douloureux); 2) 30 psychopathies, dont 11 schiz?phrénies, 8 psychopathies infantiles, 3 psychoses hallucinatoires chroniques, 3 névroses obsessionnelles, deux cœentopathies hypocondriaques, une agitation anxieuse, une manie chronique, une psychose complexe.

Nous n'avons pas l'intention d'étudier ici nos résultats thérápeutiques, malgré le caractère souvent très favorable obtenu dans de nombreux cas. Nous nous bornerons à l'étude séméiologique. Les troubles que nous étudions sont d'ailleurs surtout évidents dans les premières semaines post-opératoires. Avant de décrire leurs variations suivant la maladie en cause et suivant le type d'intervention, nous essaierons de grouper les príncipaux symptômes dans une description d'ensemble.

\section{LE SYNDROME COMMUN}

Après lobotomie, Freeman et Watts ont observé des réactions excessives aux diverses stimulations. Feuillet et Robii trouvent leurs malades "douillets", avec le sursaut fàcile, des réactions vives aux excitations cutanées désagréables. Nous avons personnellement distingué les paresthésies spontanées et les réactions provoquées.

Les paresthésies spontanées consistent surtout on démangeaisons de la peau. Le frottement du nez, déjà signalé dans les lésions préfrontales par Wartenberg, est particulièrement fréquent, mais on observe aussi le frottement de la région péribuccale, du front, du crâne (ce qui entraîne le dérangement du pansement et peut avoir de sćrieux inconvénients), des mains, de la poitrine, parmi les régions préférées.

Une de nos malades, trois semaines après une lobotomie bilatérale, nous dit spontanément, sans que jamais nous ayons attiré son attention 
sur ce point: "J'ai le nez qui me déniange". Un autre malade, plus d'un mois après une topectomie, se plaint à plusieurs reprises de ressentir des piqûres (d'abeilles, de fourmis rouges) au niveau de la tête; au cours de l'examen, il tourne brusquement la tête, persıadé que certairıs voisins lui iancent des aiguilles, ou des "instruments à cuti-réaction". Il s'agissait d'un schizophrène paranoïde et l'on ne peut contester chez lui un élément interprétatif; la réporise paranoïaque, chez ce malade, appáraît comme un complexe sensitivo-moteur immédiat, privé du contrôle mental. Dans d'autres cas cependant les paresthésies surviennent chez des sujets ne présentant pas de troubles psychiques apparents.

Les réactions provoquées par des stimulations simples, telles que le pincément, la piqûre, le chatouillement, le froid, sont généralement vives, immédiates, accompagnées de réactions de défense, et souvent associées à de: réactions émotives telles que le fou-rire ou une attitude légèrement hostile, avec des réponses verbales diverses ("lai:ssez-moi, vous m'embêtez", etc.). La pression des nerfs (cubitaux et surtout trijumeaux), des testicules chez l'homme, provoque des réactions antagonistes ou des grimaces du visage.

Chez certains malades, les stimulations paraissent provoquer des réactions érotiques; nous citerons le cas d'un sujet qui, peu après une topectomie, avait des érections au cours de l'examen et celui d'une femme (une maniaque il est vrai) qui, après lobotomie, présentait, quand on la piquair, des attouchements de la région génitale. Plus souvent il s'agit seuleinent d'une "attitude érotique", avec sourires équivoques.

Dans le premier mois post-opératoire, les malades ont souvent une peur instinctive des piqûres, avec parfois réactions de fuite, nécessiiant deux ou trois personnes pour les immobiliser. Cette peur auginente certainement les réactions mais ne suffit pas à les expliquer; si, après cvoir rassuré le malade et lui avoir dit de fermer les yeux, on produit une stimulation, on obtient une réponse immédiate, anormale par sa brusquerie, son intensité et sa diffusion. Parfois le sujet gratte avec sa main ou son nied la région irritée, durant quelques secondes, ce qui semble indiquer une persistance anormale de la sensation.

Cependant, les sensibilités élémentaires à tous les modes, le tact, la pression, la sensibilité au diapason, les sensibilités thermiques sont toujours conservées. La stéréognosie paraît grossièrement indemne, mais nous n'avons pas étudié systématiquement les altérations légères de la sensibilité discriminative.

La fréquence des troubles sensitif, après lobotomie ou topectomie bilatérale, est très grande. Les réactions peuvent être masquées par l'inertie du malade, surtout après lobotomie; mais, cette réserve faite, nous pensons que la surréactivité est un phénomène constant, survenant très tôt (car on la constate souvent dès le lendemain de l'intervention), persistant plu- 
sieurs semaines, mais s'atténuant généralement à partir de la 5ème ou 6ème semaine. Plusieurs mois après l'opération, les malades peuvent rester chatouilleux mais la surréactivité riest plus aussi évidente.

Fait surprenant, sur lequel nous reviendrons, les douleurs irréductibles sont généralement atténuées, ce qui contraste avec les signes précédents.

\section{TROUBLES SENSORIELS}

1) Troubles visuels - Très souvent, nous avons constaté une légère photophobie, avec larmoiement. L'hippus, signe sur lequel Bianchi avait attiré autrefois l'attention, est d'interprétation assez difficile car beaucoup de malades, surtout les psychopathes, présentent de l'instabilité des réactions pupillaires à la lumière. Après l'intervention, ces réactions nous ont paru plus évidentes et les examens des ophtalmologistes en font souvent mention. Du myosis, avec diminution de la réflectivité luminause, est plus rarement observé.

2) Troubles auditifs -- Le bruit est souvent mal supporté pa: les frontaux, comme l'avait signalé Dollken, et plus récemment Rylander. Après lobotomie ou topectomie, les malades ont souvent des réactions excessives au bruit, ils deviennent irrités lorsqu'ils entendent les plaintes des autres malades ou des cris d'enfants, que ceux-ci leur appartiennent ou nor. Nous n'avons pu faire étude audiométrique comparative.

3) Troubles vestibulaires et troubles de l'orientation spatiale - Les iroubles de l'orientation spatiale ont été signalés dans les lésions frontales par Pierre Marie; les recherches de L. Barraquer-Ferré en ont démontré la réalité expérimentale. Leur observation est malheureusement difficilc chez des opérés.

Avec Winter, nous avons étudié les réaciions vestibulaires; nous n'avens pu trouver que des troubles légers et variables, sans systématisation nette, tantôt de l'hyperexcitabilité tantôt de l'hypoexcitabilité. Dans les cas où le malade s'améliore, les réponses vestibulaires tendent à revenir à la normale. La variabilité des réporses est comparable à la variabilité des chronaxies vestibulaires dans les affections mentales, constatée par G. Bourguignon.

4) Hallucinations - On peut les ranger parmi les troubles sensoriels. Elles sont particulièrement fréquentes au cours des tumeurs frontales, comme le fait ressort d'une étude d'ensemble de Courville, inspirée par H. Cushing. Nous avons invoqué, à l'origine de certaines hallucinations, la suppression fonctionnelle préfrontale plus ou moins complète; pour certains cas, il faudrait plutôt, semble-t-il, invoquer un dysfonctionne- 
aen! préfrontal. Ces hallucinations de type frontal sont complexes, préscritent un caractère de réalité intense, s'associent à une surréactivité diffuse et à une dépersonnalisation.

L. Barraquer-Ferré a publié une remarquable observation d'hallucinations visuelles et olfactives au cours de lésions de sarcomatose métastatique des deux lobes frontaux. Nous avons étudié un cas très suggestif avec Borel, Peiti-Dutaillis et Janny, chez un malheureux qui, par tentative de suicide, avait réalisé une lésion orbito-frontale bilatérale. Nous avons pu ohserver quatre autres cas de délire onirique: l'un après topectomie de l'aire 9 chez un sujet atteint de psychalgie faciale, un autre après topectomie de l'aire 9 et de la circonvolution limbique, chez un grand caractériel épileplique; enfin chez deux hommes souffrant d'algies cancéreuses, après lobotomie bilatérale. Le Beau signale deux cas de délire onirique après topectomie. Krayenbuhl et Sroll décrivent une observation d'état hallucinatoire prolongé, associé à un état catatonique, après lobotomie.

On peut évidemment nous objecter que ces phénomènes oniriques, survenant dans la première semaine post-opératoir ¿, peuvent être causés par de l'œdème cérébral; mais, à vouloir tout expliçuer par l'œdème ou toute autre cause générale hypothétique, on risque une "auto-anesthésie" ("selfnarcotization"), comme le fait spiritucllement remarquer Mettler, susceptible de faire négliger tout l'intérêt dıs observations.

\section{TROUBLES AFFECTIFS}

Nous ne ferons que rappeler ici les réactiors affectives bien connues au cours des lésions frontales, en particulier la classique euphorie, très fréquente mais non courante.

L'incontinence émotionnelle, suivant l'expression de Rylander, est un signe majeur qu'on observe surtout dars les deux premiers mois post-opératoires; elle se manifeste par de petits signes tels que le fou-rire non motivé, par l'impossibilité de dissimuler ses émotions, que celles-ci soient de caractère agréable ou pénible. Cette incontinence peut déterminer des réactions paradoxales. Une de nos malades, après lobotomie unilatérale ¿auche, pour algies cancéreuses, disait, quand on lui retirait le bassin: "C'est du vrai Champagne". Un lobotomisé bilatéral, également pour algies cancéreuses, ne pouvait plus supporter la vue ni les plaintes de son voisin de lit, un nord-africain, et faisait des remarques grossières ̀̀ son sujet; à la vue d'une infirmière, il fit sans aucun tact allusion aux grains de beauté de ses bras. Si nous signalons ces détails, c'est parce qu'on retrouve, là encore, le défaut d'inhibition, la réponse brusque et immédiate qui, nous l'avons vu, caractérise les réponses sensitivo-sensor:elles de ces sujets. 


\section{VARIATIONS SYMPTOMATIQUES}

On peut distinguer des modalités réactionnelles paraissant dues à l'état antérieur du sujet ou au type d'intervention.

\section{A - Suivant l'état antérieur du sujet:}

1) Au cours des algies irréductibles, on peut s'étonner de la sédation apparente des douleurs, car elle coexisie avec une surréactivité sensitivosensorielle. Comme l'ont remarqué différents auteurs, Freeman et Watts en particulier, ces sujets ne paraissent plus obsédés par leur douleur, ne la ressentent plus qu'en tant qu'expérience passagère; certains s'en plaignent encore si on les interroge à ce propos, d'autres ne souffrent plus et s'en étonnent; tel malade qui réclamait la morphine à grands cris n'en fait plus mention.

Chez tous nos algiques, nous avons obtenu une sédation, au moins partielle et transitoire des douleurs. On peut se demander si cette sédation n'est qu'apparente, car les réponses de ces sujets sont souvent paradoxales. Chez certains opérés nous avons observé une discordance dans les réponses. Ainsi une malade, à qui avait été faite une ablation des aires 9 pour soulager des algies pelviennes d'origine cancéreuse, avait des grimaces douloureuses du visage puis, aussitôt après, si on l'interrogeait, disait "tout va bien".

Les réponses peuvent avoir un caractère ambivalent, le sujet affirmant tantôt qu'il souffre, tantôt qu'il ne souffre pas. Ces discordances compliquent l'interprétation des résultats. Cependant l'aspect du visage plus détendu, les périodes d'euphorie entre les accès douloureux constituent un mieux. L'intervention a modifié le mode réactionnel à la douleur, les malades extériorisent davantage leur souffrance par des grimaces, par des cris ou en agrippant ce qui est à leur proximité. Comme nous le disait une infirmière, ils paraissent souffrir "d'une maniere plus animale".

Sans vouloir ouvrir un débat philosophique sur la douleur comparée chez l'animal et chez l'homme, on doit reconnaîtye que, chez des malheureux voués à une fin atroce, on a le sentiment d'amoindrir la souffrance. Douleur sur un mode plus animal sans doute, et aussi plus puéril, plus instinctif: une opérée, âgée de 38 ans, nous réclamait sa mère et, bien que la sachant décédée depuis 1929, se demandait comment on pourrait la faire venir. On ne saurait méconnaître que ces modalités réactionnelles créées par la psycho-chirurgie vont de pair avec une indifférence idéo-affective, une abolition du côté sublime de la souîfrance. Mais certaines douleurs outrepassent la tolérance humaine et cette considération nous paraît justifier l'intervention.

La durée de la sédation douloureuse est difficile à préciser: nos quatorze malades atteints d'algies d'origine néoplasique étaient de grands ma- 
lades, condamnés à une fin prochaine. Certains inoururent dans le service, de leur maladie cancéreuse, d'autres furent ramenés dans leur familile. Nous avons eu plutôt l'impression que l'intervention aggravait la maladie et on peut se demander si, en rajeunissant l'individu, la libération préfrontale n'augmente pas le potentiel évolutif des tumeurs. Il y a là un problème que pourrait peut-être résoudre l'expérimentation animale.

2) Dans les maladies mentales, nous avons également observé des phénomènes de surréactivité sensitive et sensorielle. Mais l'état antérieur du sujet modifie les réactions.

Les schizophrènes, après lobotomie ou topectomie, traversent parfois une phase d'inertie profonde, avec bizarreries d'attitudes, mutisme, et la surréactivité apparaît d'emblée après l'intervention, d'où un contraste remarquable avec l'aspect antérieur. Nous avons observé trois schizophrènes hallucinés: dans les trois cas, les hallucinations furent évidentes dans la période post-opératoire; chez deux malades, elles persistaient plusieurs mois après l'intervention, peut-être avec une moindre charge émotive; chez le troisième malade, atteint d'hallucinations religieuses, elles s'effacèrent à partir de la troisième semaine post-opératoire et n'ont pas réapparu, aux dires du malade, durant les sept mois suivants, délai d'observation.

Dans trois psychoses hallucinatoires chroniques, nous avons vu s'estomper peu à peu et finalement disparaître le syndrome d'influence, les hallucinations ayant disparu dans deux cas.

Il est certain que chaque malade réagit suivant son état antérieur, avec ses caractéristiques psycho-affectives. Le schizophrène garde le mode de pensée propre à cette maladie, l'obsédé conserve en lui les germes de sa névrose. Mais nous estimons que la création de nouveaux automa. tismes sur les plans sensitif, sensoriel, affectif et instinctif, bien qu'aboutissant à des réponses plus élémentaires, est souvent préférable à la complexité, à l'anarchie causées par la maladie antérieure.

B - Suivant le type d'intervention, on peut distinguer des variations dans le tableau symptomatique. D'une manière générale, l'inertie psychomotrice est peut-être plus grande et prolongée après une lobotomie hilatérale qu'après une topectomie étendue, si on compare des malades atteints de la même affection. Les effets d'une topectomie étendue aux aires 9,10 et 46 sont plus marqués et surtout plus durables que ceux d'une topectomie limitée aux aires 9.

Après lobotomie unilatérale, le syndrome sensiiif et sensoriel est souvent très discret et peu durable, les troubles mentaux minimes en apparence; cependant dans les cinq cas où cette opération a été pratiquée (quatre fois du côté gauche et une fois du côté droit), nous avons ašsisté à une amélioration nette des algies, au moins transitoire. 
Le syndrome sensitivo-moteur que nous avons décrit nous paraît traduire une libération thalamique et vraissemblablement du paléo-thalamus, par l'intermédiaire des noyaux dorso-médians, eux-mêmes reliés avec le système neuro-végétatif périventriculaire ei avec le pallidum. Cette hypothèse, défendue par nous en 1939, est en conformilé avec les faits anatomiques et a trouvé sa confirmation dans la plupart des recherches récentes. La suppression préfrontale aboutit notamment à la libération des formes élémentaires de la sensibilité, correspondant à la sensibilité dite protopathique de $\mathrm{H}$. Head. On nous objectera que le syndrome thalamique comporte des douleurs spontanées, ce que nous n'avons pas observé chez nos malades; mais ces douleurs, suivant différents auteurs, et comme y insiste Lhermitte, paraissent dues à l'irritation de certains noyaux, et non à leur libération.

Les hallucinations, les états oniriques peuvent être expliqués par la libération du système neuro-végétatif (qui, suivant la conception de Mourgue, joue un rôle fondamental dans leur genèse), à la faveur d'une suppression fonctionnelle préfrontale. Cependaut, lorsqu'une intervention supprime des hallucinations préalables, on peut se demander si elle n'a pas agi en améliorant un dysfonctionnement hypothalamo-thalamo-préfrontal.

On ne saurait dissocier le syndrome sensitivo-sensoriel des autres signes traduisant une libération extra-pyramidale (hypotonie ou hypertonie discrète, tendance cataleptique, stéréotypies, modifications de l'activité) si souvent constatées après lobotomie ou topectomie. Nous avons très souvent constaté, à partir de la 2ème ou 3ème semaine post-opératoire, l'exagération des réflexes médians (en totalité ou pour certairs) et notamment du naso-palpébral qui diffuse au muscle mentonnier. Cette exagération est aussi un indice de libération extra-pyramidale.

Les modifications neuro-végétatives, les engraissements paradoxaux sont actuellement bien connus et les travaux récents de Rinkel, Greenblatt et de leurs associés ont démontré la libération du système neuro-végétatif après lobotomie. Nous avions personnellement, dès 1937, montré que l'ablation préfrontale chez le singe permet d'observer une exagération des réponses, aussi bien orthosympathiques que parasympathiques.

La création de nouveaux automatismes sensitivo-moteurs peut aussi, à notre avis, justifier les troubles de l'attention, de la mémoire, la distractibilité de ces malades, par défaut d'intégration corticale. Elle permet un jeu intellectuel si le fonds mental est suffisant mais ce jeu, assurément, perdra en profondeur.

Cette théorie est en conformité avec la thèse de dissolution du système nerveux de H. Jackson, mais à condition de mettre l'accent sur le travail de réparation et de compensation de l'oıganisme grâce à la syneidesis 
de von Monakow et Mourgue, c'est à dire à l'équilibre des diverses vàleurs instinctives.

Eile rejoint la théorie de dissolution-reconstruction (suivant l'appella. tion de Delmas-Marsalet), invoquée par Feuillet et Robin pour expliquer l'effet des lobotomies et permet de comparer cet effet à celui des électrochocs, conformément à la conception de Delay et Soulairac. Le terme de syntonisation régressive, adopté par Barahona Fernandes, est particulièrement explicite, dans le même ordre d'idées.

Le retentissement de la psycho-chirurgie sur la douleur reste assez mystérieux: suivant notre hypothèse, on peut évidemment invoquer un défaut d'intégration corticale de la douleur mais on est surpris de voir coïncider une sensibilité protopathique exaltée avec une amélioration apparente.

Il est possible que les modifications neuro-végétatives soient en cause, si l'on tient compte de l'importance du système neuro-végétatif dans la douleur physique, conformément aux idées de Leriche.

Tout aussi difficile est l'interprétation des résultats dans les maladies mentales. Le mieux qu'on puisse dire, en l'état aciuel de nos connaissances, c'est qu'on libère certains automatismes sensori-moteurs de l'influence néfaste exercée par un dysfonctionnement préfronto-thalamo-hypothalamique.

\section{BIBLIOGRAPHIE}

1. Barahona Fernandes, H. J. - Anatomo-physiologie cérébrale et fonctions psychiques dans la leucotomie-préfrontale. Rapport présenté au Congrès Internat. de Psychiat., Paris, 1950.

2. Barraquer-Ferré, L. - Contribution à l'étude de la physio-pathologie et de la séméiologie des lobes frontaux. Editorial de Publicaciones Médicas, 1946.

3. Borel, A., Petit-Dutaillis, D., Messimy, R. et Janny, P. - Délire onirique avec fabulation fantastique, consécutif à une lésion orbito-fiontale par projectile. Rev. Neurol., 81:300-303, 1949.

4. Bourguignon, G. - La chronaxie vestibulaire dans les psychoses. Ann. Médico-Psychol., $n^{\circ}$ 3, Mars, 1948.

5. Chapman, W. P., Rose, A. S. et Solomon, H. C. - Measurements of heat stimulus producing motor withdrawal reaction in patients following frontal lobotomy. In: The frontal lobes. A. Res. Nerv. a. Ment. Dis., 27:754-768, 1948.

6. Delay, G. - Sur la lobotomie préfrontale. Presse Méd., 58:77-78 (28 Janvier) 1950.

7. Feld, M. et Messimy, R. - Contribution à l'étude de la topectomie. Presse Méd., 57:358-360 (20 Avril) 1949.

8. Feuillet, C. et Collin, J. - Le syndrome de lobotomie préfrontale. Bull. Méd. (Paris), 62:365-369 (21 Août) 1948.

9. King, H. E., Clausen, J. et Scarff, J. E. - Seuils cutanés pour la douleur avant et après lobotomie préfrontale unilatérale. J. Nerv. a. Ment. Dis., vol. 112, n 2 (Août) 1950. 
10. Krayenbuhl, H. et Stoll, W. - Prefrontal leucotomy and topectomy for relief of intractable pain. Rapport au IV Congrès Neurol. Internat., Paris, 5-10 Septembre 1949.

11. Le Beau, G., Feld, M. et Bouvet, M. - Sur la resection bilatérale de cortines aires préfrontales (topectomie) dans les troubles mentaux et dans les douleurs irréductibles. Kev. Neurol., 80:481-496 (Août) 1948.

12. Lhermitte, G., Ajuriaguerra, G. et Hecaen, H. - Le syndrome thalamique. stude clinique. Rappert au IV Congrès Neurol. Internat., Paris, 5-10 Septembre 1949 .

13. Messimy, R. - a) Faits expérimentaux et cliniques concernant les fonctions des lobes préfontaux. Ann. de Méd., 49:69-85, 1948. b) Les effets, chez le singe, de l'ablation des lobes préfrontaux. Rev. Neurol., $n^{\circ} 1$, pag. 1-37 (Janvier) 1939. c) Les effets chez l'homme des lésions préfrontales. Ann. dz Méd., 45 (Mai) 1939.

14. Mettler, F. A. et col. - Selective partial ablation of the frontal cortex. Paul B. Hoeber, New York, 1949.

15. Monakow, C. $\mathbf{V}$. et Mourgue, R. - Introduction biologique à l'étude de la neurologie et de la psychopathologie. F. Alcan, Paris.

16. Petit-Dutaillis, D., Messimy, R. et Guiot, G. -- Effets d'une leucotomie préfrontale sur le comportement d'une obsédée. Rev. Neurol., 82:57-60 (Janvier) 1950.

17. Petit-Dutaillis, D., Messimy, R. et Feld, M. - Sur les troubles sensitifs et sensoriels observés après lobotomie on topectomie préfrontale. Comptes Rendus du IV Congrès Neurol. Internat., vol, 3, Masson et Cie., F́lit., 1949, pag. 143-146.

18. Rinkel, M., Greenblatt, M., Coon, G. P. et Solomon, M. C. - Relations du l. be frontal et du système nerveux autonome chez l'homme. Arch. Neurol. a. Psychiat., 58:570-582 (Novembre) 1947.

19. Rose, A. S. - Some observations on lobotomized patients based upon routine neurologic examinations. J. Nerv. a. Ment. Dis., 109:201-209 (Mars) 1949.

20. Winter, R. et Messimy, $\mathbf{R}$. - Recherche des troubles de l'équilibration et étude des épreuves vestibulaires dans les lésions frontales occasionnées par la psycho-chirurgie (note préliminaire). À paraitre, Rev. d'Oto-Neuro-Ophtalmol., 1952.

Rue Nicolo, 44 - Paris (XVIe) - France. 